\title{
Unterstützung der internationalen Agrarforschung durch die Schweiz
}

Christina Grieder and Barbara Becker

\section{OpenEdition}

1 Journals

Electronic version

URL: http://journals.openedition.org/sjep/872

DOI: 10.4000/sjep.872

ISSN: 1663-9677

Publisher

Institut de hautes études internationales et du développement

Printed version

Date of publication: 1 mai 2002

Number of pages: $49-64$

ISSN: $1660-5926$

\section{Electronic reference}

Christina Grieder und Barbara Becker, « Unterstützung der internationalen Agrarforschung durch die

Schweiz », Schweizerisches Jahrbuch für Entwicklungspolitik [Online], 21 | 2002, Online erschienen am: 10 September 2012, abgerufen am 08 September 2020. URL : http://journals.openedition.org/sjep/ 872 ; DOI : https://doi.org/10.4000/sjep.872 


\section{UNTERSTÜTZUNG DER INTERNATIONALEN AGRARFORSCHUNG DURCH DIE SCHWEIZ}

\section{WELCHE BEDEUTUNG HAT DIE AGRARFORSCHUNG FÜR DIE ENTWICKLUNGSZUSAMMENARBEIT?}

Die FAO erwartet nach ihrem Bericht The State of Food and Agriculture (FAO, 2000) eine Stabilisierung der Weltbevölkerung bei 12 Milliarden Menschen in der zweiten Hälfte des 21. Jahrhunderts, ungefähr doppelt so gross wie im Jahr 2000. Damit Hunger und Mangelernährung bis dahin zum Verschwinden gebracht werden können, muss die Nahrungsproduktion verdreifacht werden. Seit 1997 stellt die FAO aber eine Verlangsamung in der landwirtschaftlichen Produktivität fest, 1999 wurde die niedrigste Wachstumsrate seit 1972 in der landwirtschaftlichen Produktion in den Entwicklungsländern beobachtet. Kurzfristig sieht die FAO für die Ernährungssicherheit weniger ein technisches, Umwelt- oder Demographieproblem, sondern die nicht angepassten Produktionsformen/-mittel der ärmsten Bauernfamilien, welche nicht einmal ihre eigenen Ernährungsbedürfnisse decken können. Wesentlich sei auch die ungenügende Kaufkraft der ländlichen und städtischen Armen. Die Armut der nicht landwirtschaftlich tätigen Bevölkerung sei eine direkte Folge der ländlichen Armut und der Landflucht. Längerfristig aber sieht die FAO eine neue grüne Revolution als unabdingbar für die (ressourcen)ärmsten Bauernfamilien, dies vor allem auch durch die Förderung von bisher vernachlässigten landwirtschaftlichen Kulturpflanzen (doubly green revolution).

Die FAO stellt fest, dass Investitionen in die angewandte landwirtschaftliche Forschung, landwirtschaftliche Beratung und ländliche Schulen vital waren und sind für die ländliche Entwicklung aller nationalen Ökonomien auf dieser Welt. Diese Beobachtungen werden durch neueste Untersuchungen des IFPRI ${ }^{1}$ bestätigt, nach denen Investitionen in Bewässerung, landwirtschaftliche Forschung, Landstrassen, Trinkwasser und Schulen den grössten Beitrag zur Überwindung von Hunger und Unterernährung leisten. IFPRI kalkuliert, dass die Entwicklungsländer mit jährlichen Budgetausgaben von 4,9\% für diese fünf Bereiche die Zahl der unterernährten Kinder bis zum Jahr 2020 um 44\% gegenüber 1997 senken könnten. Dies entspricht Gesamtinvestitionen von umgerechnet ca. 35 Milliarden US-\$ pro Jahr (Rosegrant et al., 2001).

Institutionelle Reformen ohne Investitionen in diese öffentlichen Güter (public goods) produzieren kein Wachstum in der Landwirtschaft. Die Wechselbeziehungen zwischen öffentlichen Investitionen in die genannten öffentlichen Güter

* Spezialistin im Bereich Landwirtschaft und Agrarforschung, DEZA.

** Geschäftsleiterin des ZIL.

1. International Food Policy Research Institute mit Sitz in Washington, eines der Future-Harvest-Zentren der CGIAR; <www.ifpri.org>. 
sind in der Darstellung $1 \mathrm{zu}$ sehen. Sie zeigt die Bedeutung der öffentlichen Investitionen in die Beratungs- und Bildungssysteme für die Armutsbekämpfung und die Ernährungssicherheit, aber auch, dass sich die Investitionen der öffentlichen Hand in die landwirtschaftliche Forschung dann entsprechend auszahlen. Sie wirken indirekt auch stimulierend auf andere Bereiche der Forschung und auf solche, die durch andere Partner durchgeführt werden (Darstellung 2 und 3). Trotzdem weist der Trend aber in eine andere Richtung: Die öffentliche Entwicklungshilfe im Bereich Landwirtschaft hat seit den 90er Jahren bis heute stetig abgenommen.

\section{Darstellung 1: Verbesserung der Produktivität in Abhängigkeit der Technologieentwicklung}

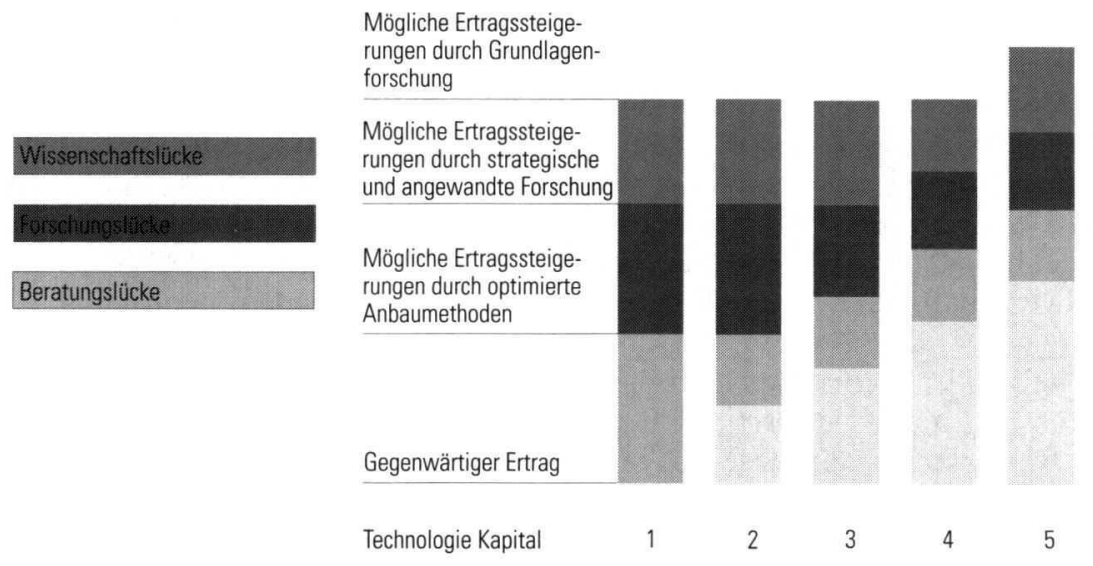

Quelle: FAO, State of Food and Agriculture 2000, S. 251.

Anmerkung: BP-A: Die Beratungslücke; sie ist die Differenz zwischen dem Ertragspotential durch eine optimierte Anbauweise und dem gegenwärtigen Ertrag. Beratungsprogramme können dieses Potential erschliessen.

RP-BP: die Forschungslücke; sie ist die Differenz zwischen dem Ertragspotential durch strategische und angewandte Forschung und optimierten Anbaumethoden. Die erfolgreiche angewandte und strategische Forschung erschliesst dieses Potential.

SP-RP: die Wissenschaftslücke; sie ist die Differenz zwischen dem Ertragspotential durch Grundlagenforschung und der strategischen und angewandten Forschung. Um diese Lücke zu schliessen, müssen internationale und nationale Basis - Forschungsprogramme neue wissenschaftliche Erkenntnisse an die strategische und angewandte Forschung liefern.

In der TK1-Ebene befinden sich die Länder, die wenig bis nichts für Beratung, Wissenschaft und Forschung tun und wo die Forschung, die für andere Regionen Technologien erzeugt, keine Beiträge zur Lösung von Problemen für diese Länder oder Region hervorbringt. In der Praxis werden die meisten Fortschritte in Regionen erzielt, die bereits die TK2-Ebene erreicht haben. Während der grünen Revolution befanden sich die Länder in Südasien auf der TK2-Stufe und haben entsprechend vom Fortschritt in der Forschung profitiert und den Sprung in die TK3-Stufe geschafft. Heute sind verschiedene Länder Südasiens und Lateinamerikas nur noch wenig von der TK4-Stufe entfernt.

Die nachhaltige Verbesserung der landwirtschaftlichen Produktivität für Kleinbauern als wirksamster Beitrag zur Armutsbekämpfung fordert auch IFAD ${ }^{2}$ im Rural Poverty Report 2000. In den ärmsten Ländern (too many people are too poor) müsse dies mit redistributiver Ermächtigung (through higher shares,

2. International Fund for Agricultural Development, Rome. 
access and control of appropriate assets, institutions, technologies and markets) einhergehen. Über die Hälfte der Ärmsten der Welt sind auf die Landwirtschaft und Landarbeit und damit auf deren dynamische Entwicklung angewiesen. Die notwendige Unterstützung durch öffentliche Zuwendungen in diesen Bereich ist aber in den Jahren 1987-98 in absoluten Werten um über zwei Drittel zurückgegangen. Darin eingeschlossen sind Investitionen für Schulen, ländliche Infrastruktur, Gesundheit, Technologieentwicklung, Forschung, institutionelle Entwicklung und Marktzugang. Effektive Armutsbekämpfung müsse deshalb Mittel wieder vermehrt in die ländliche Entwicklung leiten, fordert daher IFAD in seinem Bericht.

\section{Darstellung 2: Rolle der öffentlichen, nationalen und internationalen Forschung*}

Beziehung zwischen öffentlichem und privatem Sektor in verschiedenen geographischen

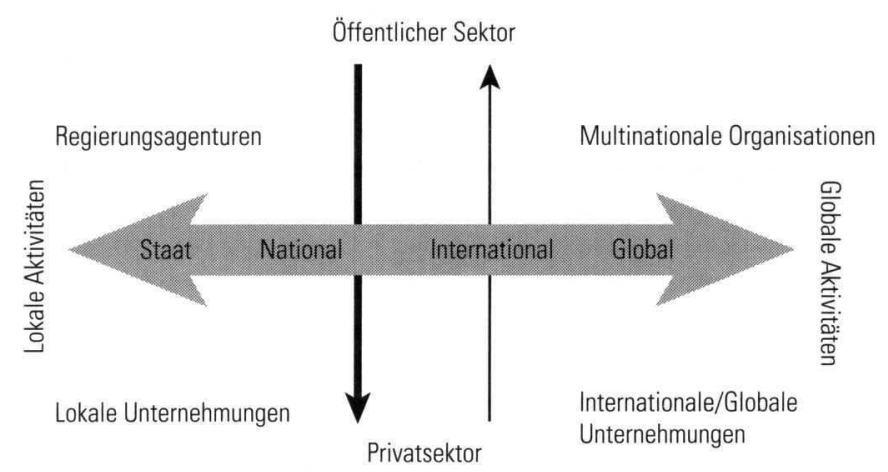

Dimensionen bei der Erzeugung von neuen wissenschaftlichen Erkenntnissen

Quelle: Dalrymple (2001).

Anmerkung: Es wird angenommen, dass jeder Quadrant mit jedem anderen in Beziehungen steht. Dabei wird aber festgestellt, dass der private Sektor meistens mehr vom öffentlichen Sektor profitiert als umgekehrt. Allerdings stimmt diese Feststellung nur noch zu einem bestimmten Grad, da der Privatsektor in gewissen wissenschaftlichen Bereichen immer wichtiger wird und zunehmend Rechte des geistigen Eigentums besitzt.

* Die verschiedenen internationalen Agrarforschungszentren sind als globale Akteure im Quadranten rechts oben anzusiedeln.

Die Weltbank schreibt in ihrem Weltentwicklungsbericht 2000/2001 Attacking Poverty, dass Investitionen des öffentlichen Sektors gegen Armuts- und Infektionskrankheiten und für die Erhöhung der landwirtschaftlichen Produktivität die wichtigsten internationalen öffentlichen Güter für die Ärmsten erzeugen. Sie sehen daher Aktivitäten in diesen Bereichen als am wirksamsten für die Bekämpfung der Armut an. Die Privatwirtschaft ist in der Erzeugung nationaler oder internationaler öffentlicher Güter in der Landwirtschaft oder Gesundheit weitgehend abwesend, weil Anreize dafür fehlen. Deshalb spielen - neben der Zusammenarbeit der Länder in diesen Bereichen - die Investitionen öffentlicher Gelder eine bedeutende Rolle, um die Armut wirksam zu bekämpfen. Die Weltbank fordert deshalb auf, wieder vermehrt Investitionen in die Bereiche Landwirtschaft und Gesundheit zu lenken.

Verschiedene Wissenschaftler haben sich in den vergangenen Jahren ebenfalls zur Rolle der landwirtschaftlichen Forschung und der landwirtschaftlichen Produktion in der Bekämpfung der Armut geäussert. Ganz wesentlich sei die Agrarforschung der CGIAR-Zentren, Erzeugerin von pro poor international public goods, stellen Ökonomen wie Jeffrey Sachs von der Harvard University oder 
Michael Lipton von der University of Sussex fest. Als besonders wichtig betrachten sie die Entwicklung und Nutzung neuer Technologien für die Ärmsten, um deren Probleme und die Ernährungssicherheit effizient und wirksam anzugehen. Beide kritisieren, dass Investitionen der Entwicklungszusammenarbeit in die Agrarforschung und in den ländlichen Raum zurückgegangen seien, obwohl der return on investment sehr hoch sei.

\section{Darstellung 3: Beziehung der öffentlichen und privaten Grundlagen- und adaptiven und angewandten Forschung und Entwicklung}

Organisation der Partnerschaften zwischen öffentlicher und privater wissenschaftlicher Forschung- und Technologieentwicklung im Quadrant Model

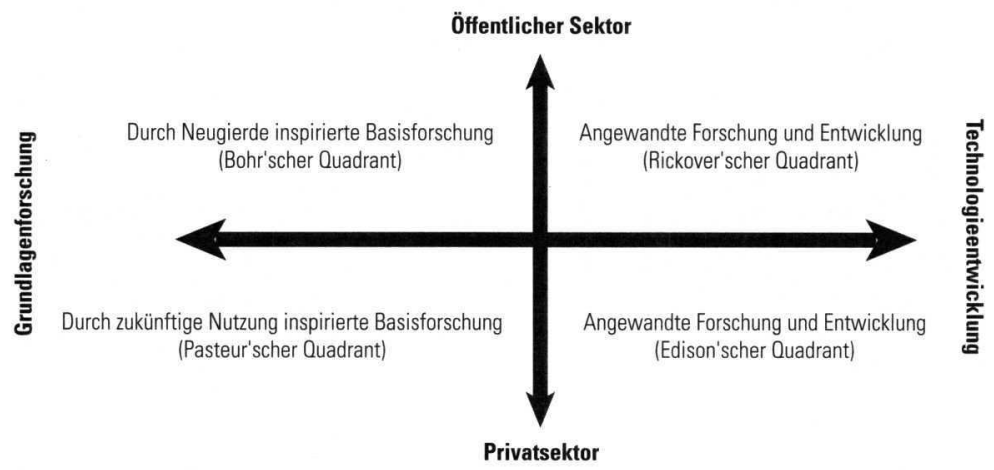

Quelle: Dalrymple (2001). Angepasst von Vernon W. Ruttan, Technology, Growth, and Development: An Induced Innovation Perspective, 0xford University Press, 2001, S. 237 (wiederum angepasst durch Ruttan von anderen Quellen).

Die Zeitspanne zwischen der Inangriffnahme von Forschungsarbeiten und der Anwendung der Ergebnisse in der bäuerlichen Praxis beträgt im Allgemeinen zwischen mehr als 10 und bis zu 20 Jahren. Die Ernährungssicherung der nächsten Generation hängt daher auch von Forschungsinvestitionen $a b$, die heute getätigt werden. Das heute rechnerisch bestehende globale Gleichgewicht zwischen Nahrungsmittelangebot und -nachfrage darf daher keinesfalls zu Selbstgefälligkeit verleiten, sondern muss durch entsprechende Investitionen in die Zukunft gesichert werden. Nach Angaben der FAO leiden heute noch immer mehr als 800 Millionen Menschen an Mangel- und Unterernährung, mehr Menschen als in Nordamerika und Westeuropa zusammen leben. Dies, obwohl heute die Bevölkerung 2,5-mal so gross ist wie in den 60er Jahren und die Zahl der Hungernden und Unterernährten sowohl in absoluten Zahlen wie in Prozenten in der gleichen Zeit gesunken ist ${ }^{3}$.

Diese beachtliche Reduktion der relativen Anzahl Hungernder ist im Wesentlichen den Erfolgen der Agrarforschung zuzuschreiben. Heute werden mehr als 300 von Future-Harvest-Zentren ${ }^{4}$ entwickelte Weizen- und Reissorten und mehr

3. FAO, The State of Food and Agriculture, 2000, p. 305.

4. Die Future-Harvest-Agrarforschungszentren der CGIAR und assoziierte Zentren wie ICIPE, etc. sind im Quadranten der Darstellung 3 rechts oben anzusiedeln. Die öffentlich finanzierte Forschung kann und soll sich auch auf Themen und Probleme konzentrieren, die nicht im Interesse der privat finanzierten Forschung sind, wie landwirtschaftliche Produktionsfragen in marginalen Gebieten und für die ressourcenarmen Bauernfamilien in Entwicklungsländern. 
als 200 Maissorten von Bauernfamilien in Entwicklungsländern neben ihren traditionellen Sorten angebaut. Die Produktivität von Weizen und Reis wurde verdoppelt. Die Weizenproduktion in Entwicklungsländern hat zum Beispiel im Wert um mehr als 1,8 Milliarden US-\$ zugenommen in den vergangenen 30 Jahren; die Reisproduktion in Indien hat sich auf der gleichen Fläche fast verdreifacht ${ }^{5}$. Die Ertragssteigerungen haben auch bewirkt, dass die Konsumentenpreise für diese Grundnahrungsmittel gefallen und für die Armen erschwinglicher geworden sind. Damit wird auch gesagt, dass die Anstrengungen der "grünen Revolution" in den 60er und 70er Jahren grosse Wirkung gezeigt haben. Die erzielte grössere Produktivität hielt mit der rasch zunehmenden Weltbevölkerung mehr als mit.

Im Bereich der Tierproduktion hat ebenfalls eine beträchtliche nachfragebedingte Steigerung stattgefunden, die erst durch Forschung und Technologieentwicklung ermöglicht wurde (vor allen auf den Gebieten der Züchtung, Tierernährung und Krankheitskontrolle, Fuglie et al. 2000). In den Entwicklungsländern stieg die Produktion tierischer Produkte in den letzten 20 Jahren durchschnittlich um über $5 \%$ für Fleisch und über $3 \%$ für Milch. Der Marktwert dieser Produktionssteigerung übersteigt den durch die "grüne Revolution" erzielten Mehrwert der Getreideproduktion (Delgado et al., 2001).

Heute betrachtet man die Erfolge der «grünen Revolution» aber auch kritisch. Die höhere Produktivität, die hauptsächlich durch Hochertragssorten im Intensiv-Anbau mit Dünger und Pflanzenschutzmitteln sowie durch die Intensivierung der Tierproduktion erreicht wurde, hatte Schäden in den Agrar-Ökosystemen zur Folge, die heute nicht mehr hingenommen werden können. Die nach wie vor erforderliche Steigerung der landwirtschaftlichen Produktivität muss deshalb ökologisch sensibler gestaltet werden, damit die Landwirtschaft für die weiter wachsende Weltbevölkerung auf nachhaltige Weise genügend Nahrung produzieren kann. Gleichzeitig soll die ländliche Bevölkerung in den ärmsten Ländern am meisten von diesen Anstrengungen der Forschung und Entwicklung profitieren und aus der grossen Armut herauskommen. Die Arbeiten der vergangenen zehn bis fünfzehn Jahre in Richtung nachhaltiger landwirtschaftlicher Produktion zeigen Wirkung. Beispielsweise wurde der Pestizideinsatz in Entwicklungsländern durch Methoden des integrierten Pflanzenschutzes stark gesenkt. Diese wurden durch Future-Harvest-Zentren, ICIPE ${ }^{6}, \mathrm{CABI}^{7}, \mathrm{FAO}$ und Forschungsinstitutionen im Norden in Zusammenarbeit mit nationalen Partnern in Entwicklungsländern entwickelt. Heute wird dank der Kontrolle der Insektenplagen im Cassava-Anbau in Afrika ein jährlicher Mehrwert von rund 400 Millionen US-\$ generiert (siehe Fussnote 5). Die Anstrengungen liegen seit den 80er Jahren auch vermehrt in der Erhaltung der pflanzlichen Vielfalt bei den Nutzpflanzen, um die Merkmale von lokalen Pflanzen zu nutzen und mit besseren Erträgen zu verbinden (zum Beispiel Trockenresistenz, Kälteresistenz, Eiweissgehalt, etc., siehe Fussnote 5). Erhöhte und stabile Erträge sollen gleichzeitig bei sinkenden Düngergaben erzielt werden (low input agriculture).

Neben der stärkeren Berücksichtigung ökologischer Belange spielt der soziokulturelle und ökonomische Kontext der Technologieentwicklung eine immer

5. <www.cgiar.org/who/wwa_impact.html>.

6. International Centre of Insect Physiology and Ecology, Nairobi, Kenia; <www.icipe.org >

7. Centre for Agriculture and Bioscience International, Wallingford, UK; <www.cabi.org $\rangle$. 
grössere Rolle in der internationalen Agrarforschung. Beispielsweise liefert IFPRI mit seinem auf ökonomischer Modellierung basierenden Programm Vision 2020 wichtige Hinweise auf Trends und Szenarien, wie sich Produktion und Nachfrage im Agrarsektor in den nächsten 20 Jahren entwickeln werden. Diese Studien zeigen u.a., wie die rasante Urbanisierung in den Entwicklungsländern insbesondere die Nachfrage nach tierischen Produkten erhöht, und welche Massnahmen erforderlich sind, diese Nachfrage $\mathrm{zu}$ befriedigen. Damit kleinbäuerliche Betriebe von dieser Nachfragesteigerung profitieren können, müssen nach diesen Untersuchungen drei Voraussetzungen erfüllt sein, nämlich

- Marktreformen mit Investitionsanreizen für die Kleinbauern und die Vermeidung von Subventionen an Grossbetriebe,

口 Institutionen, die den Kleinbauern helfen, termingerecht zu liefern und internationale Qualitäts- und Sicherheitsstandards zu erfüllen,

\ die Bereitstellung öffentlicher Güter wie Forschung, Beratung und Infrastruktur ${ }^{8}$.

Gleichzeitig mit einer erweiterten Sicht technologischer Ansätze erfolgte ein Bewusstseinswandel bezüglich der Einbeziehung unterschiedlicher Akteure. Bei den Forschungsmethoden und -inhalten wurden zunehmend partizipative Ansätze mit den Bauern und Bäuerinnen entwickelt und on-farm-Experimente in die Forschung einbezogen. Auf der forschungsstrategischen Ebene hat sich die Erkenntnis durchgesetzt, dass die Ziele der internationalen Agrarforschung gemeinsame Anstrengungen aller betroffenen gesellschaftlichen Gruppen erfordern. Diese Erkenntnis hat zum Zusammenschluss der «Stakeholders» im so genannten Global Forum for Agricultural Research (GFAR) ${ }^{9}$ geführt, das sich den Dialog und die Zusammenarbeit zwischen den Future-Harvest-Zentren, der Privatwirtschaft, Bauernverbänden, nationalen Forschungseinrichtungen und Hochschulen sowie Organisationen der Zivilgesellschaft zum Ziel gesetzt hat. Dieser Zusammenschluss soll der Tatsache Rechnung tragen, dass alle genannten Gruppen unterschiedliche Beiträge zur Überwindung von Armut und Hunger leisten können (Darstellungen 2, 3).

Mit den Fortschritten auf dem Gebiet der Molekularbiologie nähern sich beispielsweise die Interessen und Methoden zwischen der privatwirtschaftlichen Züchtungsforschung in den Industrieländern und die der Future-Harvest-Zentren zunehmend an. Daraus ergeben sich neue Synergien und Potenziale zur Zusammenarbeit. Das globale öffentliche Gut, das die Future-Harvest-Zentren zum Beispiel in Form von verbesserten Pflanzensorten erarbeitet, steht auch Nutzniessern aus dem Norden offen. Insbesondere hat Nordamerika von verbesserten Sorten bei Weizen und Reis profitiert. Auch Länder anderer bilateraler Geldgeber profitieren von der internationalen Agrarforschung und Entwicklung. Investitionen in die Future-Harvest-Zentren fördern daher indirekt auch die eigene landwirtschaftliche Entwicklung (siehe Darstellung 2). Für die DEZA sind die CGIARForschungsprogramme wegen ihrer Brückenfunktion zwischen der Forschung im Norden und den Programmen der Entwicklungsländer sowie wegen ihrer Funktion bei der Förderung der Süd-Süd-Zusammenarbeit strategisch wichtig.

8. <www.ifpri.org/who/wwa_impact.html>.

9. GFAR <www.egfar.org> betreibt ein Sekretariat bei der FAO zur Koordination der weltweiten Aktivitäten, die in 6 regionalen Foren organisiert ist. 
Ausgelöst durch ihre Kritik an der «grünen Revolution» wurden die Nichtregierungsorganisationen inzwischen zu Partnern in der internationalen Agrarforschung, deren Stimme zunehmend an Gewicht gewonnen hat. Insbesondere die Einbeziehung lokalen Wissens und traditioneller Technologien, Methoden des ökologischen und integrierten Landbaus, und die Fokussierung auf kleinbäuerliche Betriebe wird durch diese Vertreter der Zivilgesellschaft gefordert und unterstützt. Durch die Zusammenarbeit mit diesen Gruppen wird eine stärkere Integration zwischen lokalem und globalem Wissen sowie die Vernetzung zwischen angewandter und strategischer Forschung (Darstellungen 2, 3) erreicht.

Seit einigen Jahren steht vor allem die Entwicklung in Afrika südlich der Sahara im Brennpunkt der internationalen Agrarforschung. Im Jahr 2000 sind $42 \%$ der Gesamtinvestitionen der Future-Harvest-Forschung dorthin geflossen. In den ersten 20 Jahren der CGIAR sind ungefähr $54 \%$ der Forschungsausgaben in die Verbesserung der landwirtschaftlichen Produktivität geflossen. Diese sind seit den frühen 90er Jahren auf 36\% gesunken. Entsprechend mehr Investitionen werden seither in die Bereiche Umwelt, Biodiversität und Politikforschung getätigt. Seit 1972 sind ausserdem $21 \%$ der Gesamtinvestitionen der FutureHarvest-Zentren in den Aufbau von Forschungskapazitäten und die Stärkung der NARIs ${ }^{10}$ in Entwicklungsländern geflossen.

Die Rolle der NARIs hat sich in diesen Jahren stark differenziert. Während in einigen (Schwellen)Ländern (z.B. Brasilien, China, Indien) hochentwickelte Forschungsinstitutionen mit qualifiziertem Personal verschiedene Bereiche der Agrarforschung abdecken, sind andere Länder mit grossen Problemen durch zu geringe Investitionen, Humanressourcen und Ausrüstung konfrontiert, vor allem in den Ländern Afrikas südlich der Sahara. Daraus resultieren sehr unterschiedliche Forschungspartnerschaften mit diesen Akteuren.

\section{DER BEITRAG DER SCHWEIZ ZUR INTERNATIONALEN AGRARFORSCHUNG}

Der Beitrag der Schweiz zur internationalen Agrarforschung ist seit vielen Jahren eine wichtige Säule der Schweizer Entwicklungszusammenarbeit. Diese basiert auf mehreren Säulen bezüglich der Forschungspartner sowie der beteiligten Institutionen der Forschungsförderung. Entsprechend vielfältig sind die Ziele, Inhalte, die Zielgruppen und die Formen der Zusammenarbeit (vgl. Darstellung 4). In der Schweiz beteiligen sich sowohl Förderinstitutionen als auch Forschungseinrichtungen an dieser globalen Aufgabe und tragen durch (Mit)Finanzierung, Politikberatung und -dialog und Technologieentwicklung zu den gemeinsamen Zielen der Armutsbekämpfung, Ernährungssicherung und Erhaltung der Umwelt bei. Wichtigster Träger dieser Aktivitäten in der Schweiz ist dabei die Direktion für Entwicklung und Zusammenarbeit (DEZA). 


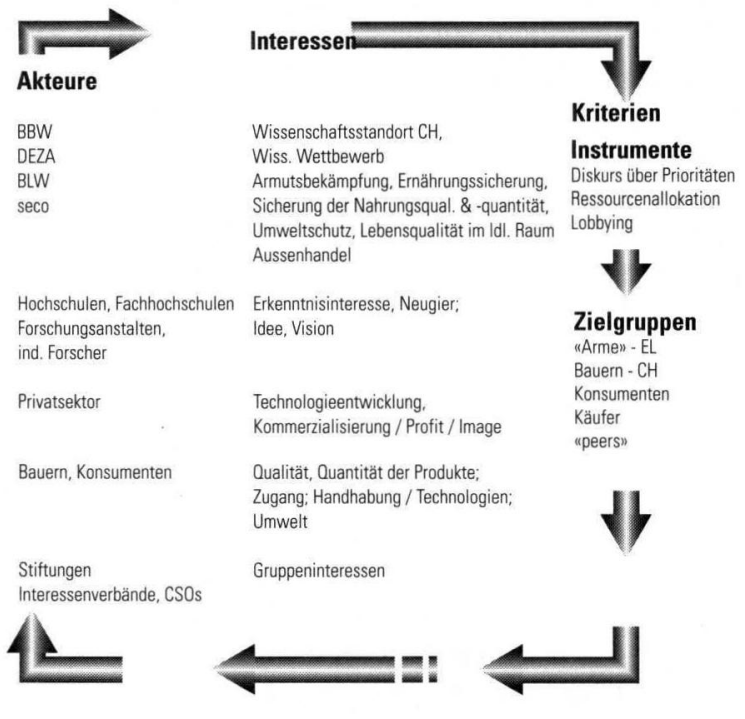

Quelle: Becker (2001).

\section{DIE INVESTITIONEN DER DEZA IN DIE INTERNATIONALE AGRARFORSCHUNG}

Wegweisend für die Arbeit der DEZA steht das Bundesgesetz über die internationale Entwicklungszusammenarbeit und humanitäre Hilfe (1976) und das 1994 vom Bundesrat verabschiedete Leitbild Nord-Süd. Im ersten Dokument wird der Auftrag der DEZA zur Bekämpfung der Armut mit Fokus ländlicher Raum in Entwicklungsländern beschrieben, im zweiten der schonende Umgang mit natürlichen Ressourcen als wichtiges Ziel festgelegt. In der Strategie 2010 der DEZA, die Mitte 2000 fertig gestellt wurde, bildet die nachhaltige Nutzung natürlicher Ressourcen auch in Zukunft einen der prioritären Bereiche. Die Verpflichtungen der Schweiz für die internationale Zusammenarbeit beim Welternährungsgipfel 1996 und im Rahmen der Konventionen zur Artenvielfalt, zur Klimaveränderung und zur Bekämpfung der Verwüstung werden von der DEZA als verbindliche Abkommen beachtet und umgesetzt. Diese verschiedenen Dokumente und Verpflichtungen bilden die Grundlage für das Engagement der DEZA in der internationalen Agrarforschung.

Die DEZA sieht in der Unterstützung der Agrarforschung für Entwicklungsländer eine wichtige Aktivität, um ihre in der neuen Strategie festgelegten Absichten im Thema «Nachhaltige Nutzung der natürlichen Ressourcen» zu erreichen. Für die DEZA ist die landwirtschaftliche Produktion darüber hinaus wichtig für die wirksame Einkommens- und Beschäftigungsförderung. Die DEZA-Sektorpolitik Landwirtschaft (1999) beschreibt die Abhängigkeit der Richtung der landwirtschaftlichen Entwicklung vom wirtschaftlichen und kulturellen Umfeld, den vorhandenen natürlichen und anderen Ressourcen und dem Zugang dazu durch die Bauernfamilien und dem (agrar)politischen Umfeld. Sie betont auch, dass die drei Entwicklungsziele der DEZA - die Reduktion der Armut, die Ernährungssicherheit und die nachhaltige Nutzung natürlicher Ressourcen - 
nur erfolgreich verfolgt werden können, wenn dies in kohärenter und abgestimmter Weise geschieht.

Die DEZA investiert seit den frühen 70er Jahren in die landwirtschaftliche Forschung für Entwicklungsländer. Wichtigste Partner waren damals die FutureHarvest-Forschungszentren der CGIAR. Die FAO ist ein wichtiger Partner für Politikfragen in den Bereichen integrierte landwirtschaftliche Produktion, nachhaltige Land- und Wassernutzung sowie Erhaltung, Zugang und Nutzung der Artenvielfalt. In den vergangenen zehn Jahren baute die DEZA auch eine intensivere Zusammenarbeit mit dem CABI und dem hauptsächlich in Afrika tätigen ICIPE und zunehmend auch mit Nichtregierungsorganisationen, die Forschungsfragen bearbeiten, auf. Die DEZA beteiligt sich sowohl mit ungebundenen (Betriebsmittel, Programmbeiträge) wie mit gebundenen Mitteln (Projektfinanzierung) an den Forschungszentren. Die meisten Projekte werden durch die operationellen Einheiten der DEZA in Afrika, Lateinamerika und Asien finanziert. Sie beschäftigen sich meistens mit der Weiterzüchtung und -anpassung von Kulturpflanzen wie Reis, Mais, Knollen- und Wurzelfrüchten wie Kartoffeln, Süsskartoffeln und Cassava, von Bohnen und anderen Leguminosen an schwierige Anbaubedingungen wie nährstoffarme Böden, Trockenheit, Nässe oder Kälte. In den vergangenen Jahren wurden vermehrt Projekte zur Erforschung schwieriger Anbausysteme in Hang- und Höhenlagen und für die Erhaltung der Artenvielfalt unterstützt. 2001 investierte die DEZA ca. 23 Millionen Schweizer Franken in die Agrarforschung der CGIAR, knapp die Hälfte davon ungebunden.

Ein wichtiges Kriterium für die Investitionen der DEZA in die internationale Agrarforschung für Entwicklungsländer ist die Erzeugung von globalen öffentlichen Gütern. Future-Harvest-Zentren halten unter einem Agreement mit der FAO über 600'000 Einlagen von Arten, Sorten und verwandten Wildpflanzen der landwirtschaftlichen Nutzpflanzen in ihren Genbanken für die Öffentlichkeit zugänglich. Diese sind für die Pflanzenzüchtung von unschätzbarem Wert und bilden den Kern eines internationalen Abkommens, des FAO International Undertaking on Plant Genetic Resources for Food and Agriculture. Die Konvention zur Artenvielfalt hat die Gestaltung dieses internationalen Abkommens an die FAO delegiert. In Leipzig wurde an einer internationalen technischen Konferenz 1996 ein globaler Aktionsplan zur Erhaltung und nachhaltigen Nutzung von pflanzengenetischen Ressourcen für Ernährung und Landwirtschaft verabschiedet. Die Ex-situ-Kollektionen in den Genbanken verschiedenster Länder und der Future-Harvest-Zentren bilden einen wichtigen Bestandteil dieses Aktionsplanes. Dabei steht die nachhaltige Nutzung der genetischen Ressourcen vor allem durch die Pflanzenzüchtung für die Entwicklungsländer als wichtiges Ziel im Zentrum. Die DEZA sieht deshalb die Unterstützung von verschiedenen Future-Harvest-Zentren mit ungebundenen Mitteln auch im Rahmen der internationalen Verpflichtungen der Schweiz durch die Ratifizierung der Biodiversitätskonvention, neben der grossen Bedeutung für die Ernährungssicherheit.

Mit der Biotechnologie befasst sich die DEZA im Rahmen der Agenda 21 zur Umsetzung der Konvention über die Artenvielfalt. Besonderes Gewicht wird auf Politiken, Zugang zu Technologien sowie Fragen der Ethik und der Gerechtigkeit gelegt. Die DEZA trägt zur Kapitalisierung der möglichen Vorteile der Biotechnologie und zur Reduzierung von deren sozioökonomischen und Umweltrisiken bei. Sie verfolgt dabei einen Drei-Wege-Ansatz: sie stärkt die 
Kapazitäten in der Entwicklung von Biotechnologie-Politiken, sie unterstützt die Fähigkeiten der Entwicklungsländer zur Nutzung der Biotechnologie und der Biotechnologie-Forschung und fördert Forschungspartnerschaften und den Technologietransfer.

Die Forschung des privaten Sektors hat für die Entwicklungsländer einen gewissen Nutzen, vor allem Erkenntnisse aus der Grundlagenforschung und der Biotechnologie. Die DEZA unterstützt deshalb die Anstrengungen der internationalen Agrarforschung, solches Wissen verfügbar zu machen oder zu nutzen. Durch die Zusammenarbeit mit Forschungsinstitutionen des Privatsektors wird die Patentierung ein Thema, das in der Vergangenheit keine oder nur eine untergeordnete Rolle spielte. Der Schutz des geistigen Eigentums in der Form von Sortenschutz oder Patenten bildet einen wichtigen Anreiz für die Privatwirtschaft, in die Forschung zu investieren. Die Bereitschaft der privaten Agrarforschung, mit Future-Harvest- und anderen internationalen Forschungszentren zu kooperieren, ist daher nur möglich, wenn die Konkurrenz im Norden dadurch nicht ebenfalls freien Zugang zu durch von ihnen entwickeltem Wissen und Technologien erhalten. Trotzdem ist sie aber bereit, relevantes Wissen für die Entwicklung und Anwendung in Entwicklungsländern zur Verfügung zu stellen. Damit zeichnet sich ab, dass die internationalen Agrarforschungszentren ihre Forschungsresultate aus einer Zusammenarbeit mit dem Privatsektor ebenfalls patentieren müssen ${ }^{11}$ mit dem Ziel, Entwicklungsländern Zugang zu geschütztem Wissen und Resultaten zu erhalten oder zu verschaffen. Das heisst, dass Future-Harvest- und andere internationale Forschungszentren dann in solche Abkommen einwilligen, wenn sie freie Lizenzberechtigungen für die Arbeit und Anwendung in Entwicklungsländern erhalten. Die Produkte von solchen Partnerschaften sind zwar nicht mehr globale öffentliche Güter, aber mindestens solche für die Entwicklungsländer.

Der Internationale Dienst für die Akquisition von Agro-Biotech Applikationen $(I S A A A)^{12}$ beschäftigt sich mit der Vermittlung von Technologien und unterstützt bei Fragen des geistigen Eigentums, die daraus entstehen können (Lizenzierungen). Die DEZA arbeitet seit 1996 mit dieser Organisation zusammen. ISAAA entwickelte mit dieser Unterstützung ein Netzwerk zur Wissensvermittlung weiter, das vor allem in Südostasien (Thailand, Vietnam, Philippinen, China) sehr aktiv ist. ISAAA arbeitet eng mit der privatwirtschaftlichen Agrarforschung zusammen und stellt dabei ein wichtiges Bindeglied zwischen NARIs und dem Privatsektor dar.

\section{Internationale Agrarforschungsaktivitäten in der Schweiz}

Neben der direkten finanziellen Unterstützung der internationalen Agrarforschungszentren fördert die DEZA die Zusammenarbeit der schweizerischen Agrarforschung mit Entwicklungsländern und Future-Harvest- und anderen internationalen Forschungszentren. Diese Unterstützung erfolgt auf mehreren Ebenen: Einzelne Länderprogramme fördern bilaterale Projekte, z.B. das Programm IndoSwiss Collaboration in Biotechnology in Indien; über das Zentrum für Internationale Landwirtschaft (ZIL) mit Sitz an der ETH Zürich werden Forschungskoope-

11. Bis anhin haben Future-Harvest-Zentren insgesamt weniger als 10 Patente angemeldet.

12. International Service for the Acquisition of Agri-Biotech Applications; <www.isaaa.org $>$. 
rationen mit internationalen Agrarforschungszentren durchgeführt; und im Swiss Forum for International Agricultural Research (SFIAR) wird der Dialog zwischen den verschiedenen Partnern in der Schweiz mit Interesse an internationaler Agrarforschung sichergestellt.

Im Zentrum für Internationale Landwirtschaft (ZIL) ${ }^{13}$ sind die meisten der an der ETHZ in der Agrarforschung und Lehre tätigen Institute und Wissenschaftler/innen zusammengeschlossen. Das ZIL bildet die Schnittstelle der Hochschule zur entwicklungsländerbezogenen Agrarforschung, was sich in einer Mischfinanzierung der ZIL-Aktivitäten durch ETHZ und DEZA niederschlägt. Als einzige universitäre Agrarfakultät der Schweiz nimmt die ETHZ damit eine strategisch wichtige Rolle in der Zusammenarbeit mit den internationalen Agrarforschungszentren ein.

Seit Bestehen des ZIL konzentrierte sich die ZIL-Forschung in so genannten Schwerpunkten. Das bisher umfangreichste Programm war die Forschung über Cassava, wo seit 1993 zehn Projekte mit einem Gesamtvolumen von über 2 Millionen Schweizer Franken durchgeführt wurden. Wichtige Themen waren dabei die Erarbeitung molekularbiologischer Grundlagen zur Virusresistenz, die biologische Schädlingsbekämpfung, Nährstoffversorgung, Mischanbau sowie sozioökonomische Fragestellungen zu Akzeptanz und Vermarktung. Wichtigste Forschungspartner waren und sind CIAT ${ }^{14}$ und IITA ${ }^{15}$.

Als weiteres Programm standen kleinbäuerliche Systeme der Milchproduktion und ihrer Vermarktung von Beginn an auf der ZIL-Agenda. Während sich die Aktivitäten in den ersten Jahren auf Indien konzentrierten, wo es eine langjährige Entwicklungszusammenarbeit der DEZA im Bereich der Tierproduktion gab, verlagerte sich die Aktivität in den letzten Jahren nach Ostafrika. Die Zusammenarbeit in diesem Schwerpunkt erfolgt vor allem mit ILRI ${ }^{16}$ und IFPRI ${ }^{17}$.

Die Aktivitäten zum integrierten Pflanzenschutz (IPM) sind seit 1997 in einem separaten Schwerpunkt zusammengefasst. Sie befassen sich mit den Wurzelund Knollenfrüchten Cassava und Yams sowie mit dem Nachernteschutz bei Körnerleguminosen. Neben dem Future-Harvest-Zentrum CIAT ist hier das Centre Suisse de Recherche Scientifique (CSRS) in der Elfenbeinküste ein wichtiger Forschungspartner.

Leguminosen zur Bodenverbesserung schliesslich bilden seit zwei Jahren den vierten aktuellen Schwerpunkt des ZIL, mit Untersuchungen zur Bodenverbesserung und zu den molekularbiologischen Mechanismen der Nährstoffversorgung. Diese Arbeiten werden in Zusammenarbeit mit dem ICRAF ${ }^{18}$ durchgeführt.

Die DEZA fördert über ein vom ZIL gemanagtes Nachwuchsprogramm ausserdem auch Arbeiten von Schweizer Forscherinnen und Forschern im Bereich Agrar- und Forstforschung in Entwicklungsländern. Über dieses Research Fellow Partnership Programme wird jährlich zwei bis drei Forscherinnen und Forschern die Möglichkeit geboten, als Doktorierende oder Postdocs an internationalen

13. <www.zil.ethz.ch>.

14. Centro Internacional de Agricultura Tropical, Cali, Kolumbien; <www.ciat.org $>$.

15. International Institute for Tropical Agriculture, Ibadan, Nigeria; <www.iita.org $>$.

16. International Livestock Research Institute, Nairobi, Kenia; <www.ilri.org >.

17. International Food Policy Research Institute, Washington DC; <www.ifpri.org $>$.

18. International Centre for Research on Agroforestry, Nairobi, Kenia; <www.icraf.org>. 
Agrarforschungszentren zu arbeiten. Dieses Programm steht Kandidatinnen und Kandidaten von allen Hochschulen und Forschungseinrichtungen der Schweiz offen, sofern sie ein gemeinsames Forschungsvorhaben mit einem internationalen Agrarforschungszentrum beantragen. Das Themenspektrum der bisher 15 über dieses Programm geförderten Projekte umfasst alle Bereiche der Agrarund Forstwissenschaften, von der Prioritätensetzung in der Biotechnologieforschung in ausgewählten Entwicklungsländern bis zur nachhaltigen Bewirtschaftung der natürlichen Walnusswälder in Kirgistan. Partnerzentren sind CIAT, CIFOR $^{19}$, CIMMYT $^{20}$, CIP $^{21}$, IITA, IRRI ${ }^{22}$ und ISNAR ${ }^{23}$ (ZIL 2000).

Neben diesem Schwerpunktprogramm des ZIL und der Nachwuchsförderung RFPP laufen und liefen an der Hochschule umfangreiche Forschungsarbeiten ausserhalb der finanziellen Förderung und des entwicklungpolitischen Mandats der DEZA. Diese Aktivitäten können in der Darstellung 3 den linken Quadranten Durch Neugierde inspirierte und Durch zukünftige Nützung inspirierte Basisforschung zugeordnet werden. Das beste Beispiel hierfür ist die Entwicklung des so genannten "Golden Rice», das auf die Initiative eines engagierten und überzeugten ETH-Forschers zurückgeht und inzwischen ein weltweites Forschungs- und Entwicklungsprogramm ausgelöst hat (Potrykus 2001, IRRI 2001). Gegenwärtig haben sich die Aktivitäten von der Molekularbiologie auf die Sicherung der geistigen Eigentumsrechte in der Public Domain verlagert.

Die Forschung zum «Golden Rice » ist exemplarisch für das Selbstverständnis der ETHZ, sehr grundlagennahe «High-Tech-Forschung» zu betreiben, bei der ein gesellschaftlicher Anwendungsbezug explizit erwünscht ist (Leitbild der ETHZ 1996). Gleichwohl gibt es auch an der ETHZ Forschungsaktivitäten, die deutlich näher an der Umsetzung sind, z.B. entwicklungsländerrelevante Auftragsforschung der Privatwirtschaft (z.B. Marktstudien, Produktentwicklung) oder die Begleitforschung in Projekten der Entwicklungszusammenarbeit (z.B. Forstwirtschaft).

Anwendungsnahe Forschung (Darstellung 3: Angewandte Forschung und Entwicklung) wird traditionellerweise von den Fachhochschulen wahrgenommen. In der Schweiz ist dies für die internationale Agrarforschung vor allem die Schweizerische Hochschule für Landwirtschaft (SHL) in Zollikofen, die zum Verbund der Berner Fachhochschulen gehört. Dozierende dieser Hochschule nehmen beispielsweise an Projektevaluierungen und ähnlichen Missionen im Auftrag der DEZA oder anderer Institutionen der Entwicklungszusammenarbeit teil. Die SHL betreut auch im Auftrag der DEZA den Informationsdienst infoagrar mit der Internetplattform Swissnode <www.sfiar.infoagrar.ch>, dem Online-Service des Swiss Forum for International Agricultural Research (SFIAR).

Weitere Schweizer Akteure in der internationalen Agrarforschung sind Unternehmen der Privatwirtschaft, Nichtregierungsorganisationen (NGO) und ausseruniversitäre Forschungseinrichtungen. Alle drei Gruppen sind in der rechten Hälfte von Darstellung 3 anzusiedeln, d.h. eher bei der Technologieentwicklung

19. Centre for International Forestry Research, Bogor, Indonesien ; <www.cifor.org >

20. Centro Internacional de Mejoramiento de Maíz y Trigo, Mexico City, Mexico; <www.cimmyt.org>.

21. Centro Internacional de la Papa, Lima, Peru; <www.cip.org >.

22. International Rice Research Institute, Los Baños, Philippinen, <www.irri.org $>$.

23. International Service for National Agricultural Research, Den Haag, Niederlande; <www.isnar.org >. 
als bei der Grundlagenforschung. Aus dem Bereich der Privatwirtschaft ist der wichtigste Akteur die Syngenta bzw. die Syngenta-Stiftung, die beide vor allem in den Sektoren Pflanzenschutz und Saatgut tätig sind. Bei den NGO betreibt u.a. Intercooperation Projekte der Land- und Forstwirtschaft, die z.T. Komponenten anwendungsbezogener Forschung umfassen. Unter den ausseruniversitären Forschungseinrichtungen arbeitet das Forschungsinstitut für biologischen Landbau (FibL) mit Forschungseinrichtungen in ausgewählten Entwicklungsländern (z.B. mit China im biologischen Obstanbau) zusammen.

Diese unvollständige Aufzählung soll einen Eindruck von der inhaltlichen und institutionellen Vielfalt der Forschungszusammenarbeit zwischen der Schweiz und der internationalen Agrarforschung vermitteln (vgl. IUED, 2000). Seit 1998 sind alle an der internationalen Agrarforschung interessierten Gruppen im Swiss Forum for International Agricultural Research (SFIAR) zusammengeschlossen. Dieses Forum dient dem Dialog und der Vernetzung der unterschiedlichen Interessengruppen, um die Zusammenarbeit, Synergien und Komplementaritäten zwischen den verschiedenen Akteuren der internationalen Agrarforschung in der Schweiz zu fördern und damit die Wirkung der schweizerischen Investitionen in diese Forschung und die damit verbundene Entwicklung zu verbessern. Mit der Gründung von SFIAR wird in der Schweiz der internationalen Entwicklung zur stärkeren Vernetzung der Stakeholders Rechnung getragen, die weltweit nicht nur auf nationaler Ebene, sondern auch regional und global vollzogen wird. Regional ist die Schweiz mit ihren verschiedenen Interessengruppen in das European Forum for Agricultural Research for Development (EFARD) eingebunden, weltweit in das Global Forum for Agricultural Research (GFAR).

Dieses Zusammengehen aller Akteure auf allen Ebenen ist unabdingbar angesichts der Herausforderung an die internationale Agrarforschung, zur Überwindung von Hunger und Armut beizutragen. Dass der Agrarforschung dabei eine Schlüsselrolle zukommt, zeigen alle genannten Untersuchungen übereinstimmend. Diese Aufgabe erfordert einen grossen finanziellen, technologischen und intellektuellen Einsatz. Die Schweiz ist gut gerüstet, ihren Beitrag zu dieser Aufgabe zu leisten. Sie muss sich dieser Herausforderung auch in der Zukunft mit allen Kräften stellen. 
Anhang 1: Investitionen der DEZA in die Agrarforschung 1990-2000, in Mio. Franken

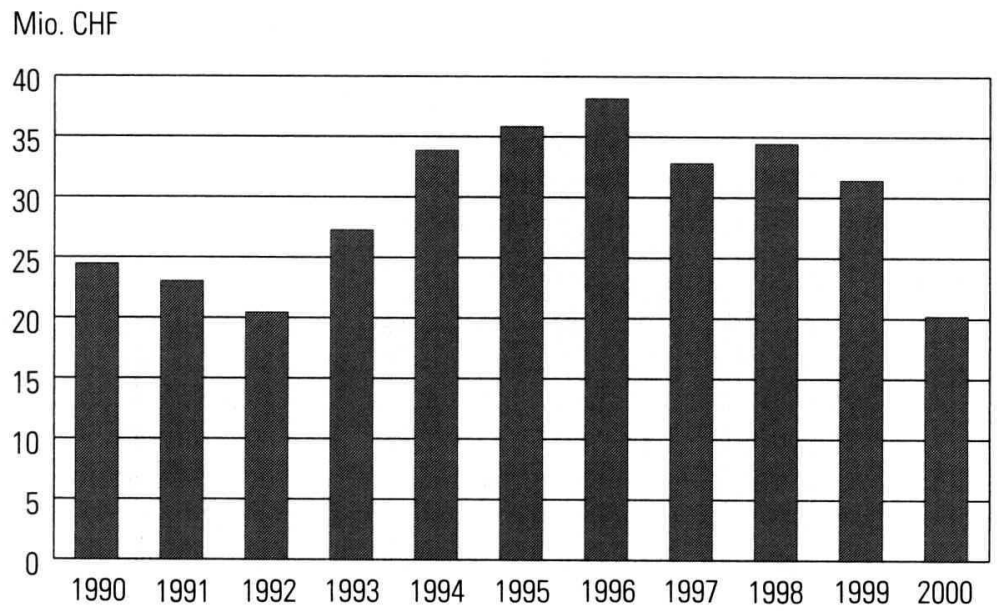

Quelle : DEZA (Catherine Graf/Christina Grieder), 2001. 


\section{Anhang 1: Investitionen der DEZA in die Agrarforschung 1990-2000 in verschiedene int. Organisationen}

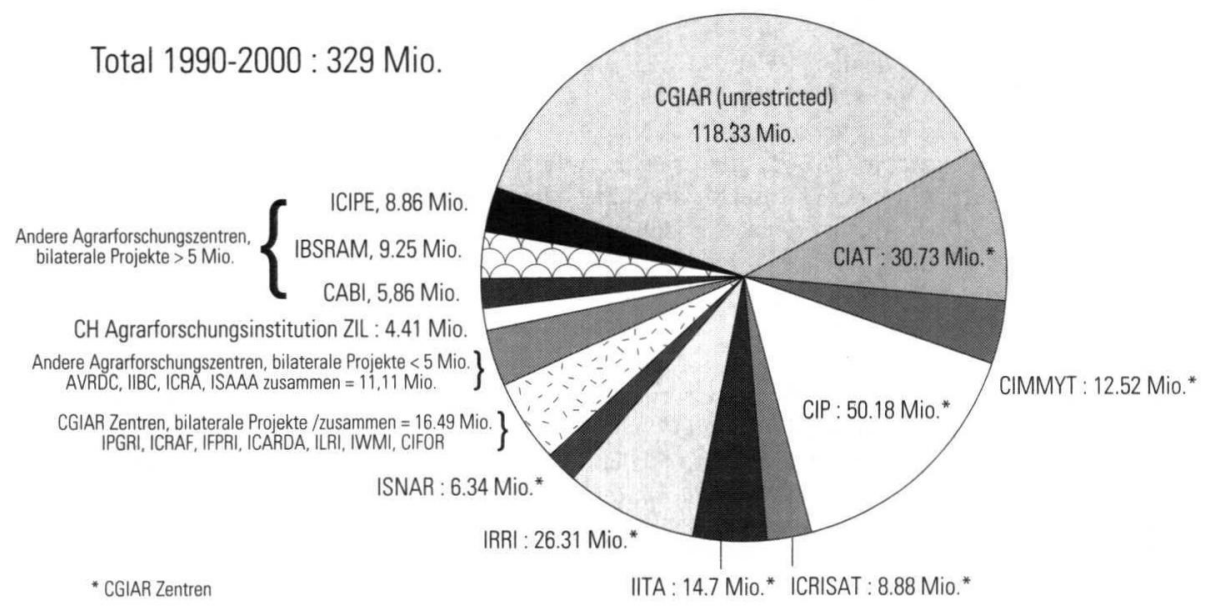

Quelle: DEZA (Catherine Graf/Christina Grieder), 2001.

AVRDC: Asian Vegetable Research and Development Center

$\mathrm{CABI}$ : Centre for Agriculture and Biosciences International

CGIAR: Consultative Group on International Agricultural Research

CIAT: Centro Internacional de Agricultura Tropical

CIFOR: Center for International Forestry Research

CIMMYT : International Maize and Wheat Improvement Center - Centro Internacional de Mejoramiento de Maíz y Trigo

CIP: International Potato Cente - Centro Internacional de la Papa

IBSRAM: International Board for Soil Research and Management

ICARDA: International Center for Agricultural Research in the Dry Areas

ICIPE: International Centre of Insect Physiology and Ecology

ICRA: International Centre for Development Oriented Research in Agriculture

ICRAF: International Centre for Research in Agroforestry

ICRISAT: International Crops Research Institute for the Semi-Arid Tropics

IFPRI: International Food Policy Research Institute

IIBC: International Institute of Biological Control

IITA: International Institute of Tropical Agriculture

ILRI: International Livestock Research Institute

IPGRI : International Plant Genetic Resources Institute

IRRI: International Rice Research Institute

ISAAA: International Service for the Acquisition of Agri-biotech Applications

ISNAR: International Service for National Agricultural Research

IWMI: International Water Management Institute

ZIL: Centre suisse pour l'agriculture internationale 


\section{REFERENZEN}

Becker, B., 2001. «Öffentliche Agrarforschung - wohin? Wie werden die Schwerpunkte in der öffentlichen Forschung gesetzt und unter welchem Druck steht die öffentliche Forschung in der Schweiz?»Vortrag bei der Tagung «Agrarforschung - für wen?» von SwissAid und Erklärung von Bern. Bern, 12.10.2001.

Bundesgesetz über die internationale Entwicklungszusammenarbeit und humanitäre Hilfe, 1976.

CGIAR, 2000, Annual Report 2000: The Challenge of Climate Change: Poor Farmers at Risk.

Dalrymple, Dana G., 2001. International Agricultural Research as Global Public Good. A Review of Concepts, Issues, and the CGIAR Experience, Entwurf 11, 26. Oktober 2001, nicht publiziert.

Delgado, Christopher L., Mark W. Rosegrant, and Siet Meijer, 2001, Livestock to 2020: The Revolution Continues. Ag-Trade, International Agricultural Trade Research Consortium. <www.agtrade.org/ digests/digest33.htm>.

DEZA, 1994, Leitbild Nord-Süd.

-, 1999, Sektorpolitik Landwirtschaft.

-, 2000, Strategie 2010 der DEZA.

ETHZ, 1996, Leitbild der ETH Zürich. <www.ethz.ch/overview/profile_de.asp>.

FAO, 2000, The State of Food and Agriculture.

Fuglie, K., C. Narrod, and C. Neumeyer, 2000. «Public and Private Investment in Animal Research», in Public-Private Collaboration in Agricultural Research: New Institutional Arrangements and Economic Implications. Eds K. Fuglie and D. Schimmelpfenning. Iowa State University Press.

IFAD, 2000, Rural Poverty Report 2000.

IRRI, 2001, "Golden Rice» Arrives in Asia. IRRI Media Hotline, 26 Jan 2001. <www.irri.org/vis/ line2001.htm\#Golden Rice>.

IUED, 2000, Jahrbuch Schweiz - Dritte Welt 2000, Genf.

Lipton, M, Reviving global poverty reduction: What role for genetically modified plants?, 1999, Sir John Crawford Memorial lecture.

Potrykus, I., 2001, «Golden Rice and Beyond». Plant Physiol, Vol. 125, S. 1157-1161, $<w w w . c h e c k b i o t e c h . o r g / r o o t /$ index.cfm?fuseaction=search\&search=rice\&doc_id $=604 \&$ start $=6>$.

Rosegrant, Mark W., Michael S. Paisner, Siet Meijer, Julie Witcover, 2001, 2020 Global Food Outlook, Trends, Alternatives, and Choices. A 2020 Vision for Food, Agriculture, and the Environment Initiative. International Food Policy Research Institute, Washington, D.C. <www.ifpri.org/2020conference/publications.asp>.

Sachs, J., «Helping the world's poorest», in The Economist, August 1999.

Weltbank, 2001, Attacking Poverty. Weltentwicklungsbericht 2000/2001.

ZIL, 2000, Annual Report 2000. Schweizerisches Zentrum für Internationale Landwirtschaft, ETH Zürich. <www.zil.ethz.ch/publications/AnnualReport2000.pdf>. 